\title{
Remote Limb Ischaemic Postconditioning Protects Against Myocardial Ischaemia/ Reperfusion Injury in Mice: Activation of JAK/STAT3-Mediated Nrf2-Antioxidant Signalling
}

\author{
Sumin $\mathrm{GaO}^{\mathrm{a}, \mathrm{e}}$ Leyun Zhan ${ }^{\mathrm{b}}$ Zhengchao Yang ${ }^{\mathrm{a}}$ Ruili Shi ${ }^{\mathrm{a}}$ Haobo $\mathrm{Li}^{\mathrm{c}}$ \\ Zhengyuan Xiac Shiying Yuan ${ }^{\mathrm{d}}$ Qing-ping Wu ${ }^{\mathrm{a}}$ Tingting Wang ${ }^{\mathrm{a}}$ \\ Shanglong $\mathrm{YaO}^{\mathrm{a}}$
}

\begin{abstract}
aDepartment of Anaesthesiology, Institute of Anaesthesiology and Critical Care Medicine, Union Hospital, Tongji Medical College, Huazhong University of Science and Technology, Wuhan, 'Department of Anaesthesiology, Three Gorges University People's Hospital, Yichang, Hubei, 'Department of Anaesthesiology, The University of Hong Kong, Hong Kong SAR, dDepartment of Critical Care Medicine, Institute of Anaesthesiology and Critical Care Medicine, Union Hospital, Tongji Medical College, Huazhong University of Science and Technology, Wuhan, e Department of Emergency Medicine, Huai'an First People's Hospital, Nanjing Medical University, Huai'an, Jiangsu, China
\end{abstract}

\section{Key Words}

Remote limb ischaemic postconditioning • JAK/STAT3 • Akt • ENOS • Nrf2 • HO-1

\begin{abstract}
Background: This study aimed to evaluate the protective effect and mechanisms of remote limb ischaemic postconditioning (RIPostC) against myocardial ischaemia/reperfusion (IR) injury. Methods: Male mice underwent 45 min of coronary artery occlusion followed by 2 $\mathrm{h}$ of reperfusion. RIPostC was achieved by three cycles of $5 \mathrm{~min}$ of ischaemia and $5 \mathrm{~min}$ of reperfusion in the left hind limb at the start of the reperfusion period. After $2 \mathrm{~h}$ of cardiac reperfusion, myocardial infarct size, cardiac enzyme release, apoptosis and oxidative stress were assessed. Protein expression and phosphorylation were measured by Western blotting. Results: RIPostC significantly decreased cardiac IR injury, as reflected by reduced infarct size and cellular apoptosis $(22.9 \pm 3.3 \%$ vs $40.9 \pm 6.2 \%$ and $13.4 \% \pm 3.1 \%$ vs $26.2 \% \pm 3.1 \%$, respectively, both $\mathrm{P}<0.01)$ as well as plasma creatine kinase-MB (CK-MB) and lactate dehydrogenase $(\mathrm{LDH})$ release $(21.97 \pm 4.08$ vs $35.86 \pm 2.91 \mathrm{ng} / \mathrm{ml}$ and $6.17 \pm 0.58$ vs $8.37 \pm 0.89 \mathrm{U} / \mathrm{ml}$, respectively, both $P<0.01$ ) compared with the IR group. RIPostC significantly increased the phosphorylation of myocardial STAT3, Akt and eNOS ( $<$-0.01). In addition, RIPostC elevated the nuclear translocation of $\mathrm{Nrf2}$ and the expression of $\mathrm{HO}-1$ and reduced myocardial oxidative
\end{abstract}

S. Gao and L. Zhan contribute equally to this work

Dr Tingting Wang and Prof Shanglong Yao

KARGER
Department of Anaesthesiology. Institute of Anaesthesiology and Critical Care Medicine, Union Hospital, Tongji Medical College, Huazhong University of Science and Technology, 1277 Jiefang Avenue, Wuhan, (China)

E-Mail wangtt201307@163.com/ysltian@163.com 


\section{Cellular Physiology Cell Physiol Biochem 2017;43:1140-1151 \\ \begin{tabular}{ll|l} 
and Biochemistry & $\begin{array}{l}\text { DOI: 10.1159/000481755 } \\
\text { Published online: October 05, } 2017\end{array}$ & $\begin{array}{l}\text { C } 2017 \text { The Author(s). Published by S. Karger AG, Basel } \\
\text { www.karger.com/cpb }\end{array}$ \\
\hline
\end{tabular}

stress $(P<0.05)$. Interestingly, pretreatment with the JAK/STAT3 inhibitor AG490 blocked the cardioprotective effect of RIPostC accompanied by decreased phosphorylation of myocardial STAT3, Akt and eNOS ( $P<0.05)$, decreased nuclear translocation of Nrf2 and expression of $\mathrm{HO}-1$, as well as increased oxidative stress $(\mathrm{P}<0.05)$. Conclusion: RIPostC attenuates apoptosis and protects against myocardial IR injury, possibly through the activation of JAK/ STAT3-mediated Nrf2-antioxidant signalling.

(C) 2017 The Author(s)

Published by S. Karger AG, Basel

\section{Introduction}

Myocardial ischaemia/reperfusion (IR) injury is the major risk during the operative and perioperative period for patients undergoing coronary artery bypass grafting (CABG) surgery. In spite of clinical improvements in myocardial protection and perioperative care, mortality is still substantial within 30 days after CABG surgery, averaging $3 \%$ to $6 \%$, and the rate of myocardial infarction remains high, up to 17.8\% [1]. Therefore, myocardial IR injury in patients with CABG is a significant challenge faced by modern cardiac practices; effective measures to attenuate myocardial IR injury may therefore improve patients' postoperative survival.

Ischaemic preconditioning and postconditioning have been widely applied to generate cardioprotection against myocardial IR injury [2-5]. However, the applicability of ischaemic preconditioning is limited by the unpredictable nature of ischaemic events during the operative period. Zhao et al. reported that local ischaemic postconditioning induced by repeated occlusion/release cycles in the left anterior descending coronary artery could reduce infarct size and preserve endothelial function in a myocardial IR model [6]. However, the local ischaemic postconditioning strategy involves repetitive trauma to the artery that may contribute to restenosis, which limits its clinical application in organs that have suffered life-threatening ischaemia. Compared with local ischaemic postconditioning, remote limb ischaemic postconditioning (RIPostC) by repeated occlusion/release cycles in the lateral femoral artery is an effective, feasible and non-invasive method to exert cardioprotection to prevent IR insult $[4,7,8]$. The cardioprotection induced by ischaemic pre-, post- and remote conditioning has shown promising results, reducing infarct size and improving clinical outcomes in patients with ischaemic heart disease. However, there have been some clinical trials showing that ischaemic pre-, post- and remote conditioning have no effect on patients with acute myocardial infarction, given that patients receiving cardiac surgery almost always have comorbidities such as diabetes, ageing, and obesity that comprise the beneficial effects of cardioprotective interventions (e.g., remote ischaemic postconditioning). It is not yet time to give up on cardioprotection through conditioning interventions; efforts should be made to further explore the underlying mechanism of these cardioprotective interventions [9, 10]. According to recent studies, the mechanism of RIPostC cardioprotection may involve autophagy [11] and transient receptor potential vanilloid 1 activation [12] in animal models. However, in patients undergoing CABG surgery, Gedik et al. reported that protection through remote ischaemic preconditioning did not appear to be associated with enhanced autophagy in left ventricular myocardium at early reperfusion [13]. Nevertheless, the underlying molecular mechanisms of cardioprotection induced by RIPostC remain poorly understood.

Recent studies have shown that the cardioprotective benefit of ischaemic postconditioning is lost in signal transducer and activator of transcription 3 (STAT3)-deficient mice, which suggests that Janus Kinase (JAK)/STAT3 activation is essential for ischaemic postconditioning [14-16]. STAT3 is important for cardiomyocyte function via modulation of mitochondrial and transcriptional responses, and, beyond that role, it also modulates the cardiac microenvironment and communicates with cardiac fibroblasts [17]. In addition, the latest study from our group has demonstrated that phosphorylation of STAT3 can activate Akt and that both play critical roles in the activation of endothelial nitric oxide synthase (eNOS) and the subsequent attenuation of myocardial IR injury [18]. Opening of the mitochondrial permeability transition pore ( $\mathrm{mPTP}$ ), which is negatively regulated by the above mentioned 


\section{Cellular Physiology Cell Physiol Biochem 2017;43:1140-1151 \begin{tabular}{l|l|l} 
and Biochemistry Published online: October 05, 2017 & $\begin{array}{l}\text { (c) } 2017 \text { The Author(s). Published by S. Karger AG, Basel } \\
\text { www.karger.com/cpb }\end{array}$ \\
\hline
\end{tabular} \\ Gao et al.: Nrf2-Antioxidant Signalling in Remote Limb Ischaemic Postconditioning Cardioprotection}

proteins, leading to abolition of mitochondrial functions, is a major mechanism of myocyte apoptosis and necrosis by IR $[19,20]$. Although many data have suggested that JAK/STAT3 plays a critical role in the cardioprotective effect of ischaemic conditioning [21], the roles of that pathway in cardioprotection induced by RIPostC remain unknown.

Excessive oxidative stress is considered one of the leading causes of myocardial dysfunction in IR injury [22]. Under physiological conditions, nuclear factor erythroid 2-related factor 2 (Nrf2) localizes in the cytoplasm and binds to its inhibitor Kelch-like ECHassociated protein 1 (KEEP1) [23]. Upon exposure of cells to oxidative stress or electrophilic compounds, Nrf2 is activated and translocates into the nucleus, where it binds to antioxidant response elements to promote a series of antioxidant genes, including heme oxygenase (HO1) and superoxide dismutase (SOD) [24]. Therefore, Nrf2 is a key component of cellular redox homeostasis in the attenuation of oxidative stress-associated pathological processes and plays an important role in cardiovascular diseases [25].

Therefore, the purpose of our study was to test the hypothesis that RIPostC protects against myocardial IR injury mainly through the activation of JAK/STAT3-mediated Nrf2antioxidant signalling in mice.

\section{Materials and Methods}

\section{Animal care}

Male C57BL/6 mice were purchased from Wuhan University (Wuhan, China) and utilized at 7-8 weeks of age. The animal protocol was approved by the Animal Care and Use Committee of Tongji Medical College of Huazhong University of Science and Technology.

\section{Animal protocol}

Mice were anaesthetized with pentobarbital sodium $(70 \mathrm{mg} / \mathrm{kg}$, intraperitoneal injection), and anaesthesia was maintained via supplemental doses of pentobarbital sodium $(30 \mathrm{mg} / \mathrm{kg})$ as needed. Tracheotomy was performed, and mice were mechanically ventilated. We induced experimental ischaemia by temporarily exteriorizing the heart via a left thoracic incision and tying an 8-0 silk suture in a slipknot around the left anterior descending coronary artery (LCA). Mice were subjected to 45 min of transitory ligation followed by $2 \mathrm{~h}$ of reperfusion.

During the reperfusion period, RIPostC was induced by 3 cycles of 5 min of left femoral artery occlusion with a microvascular clamp under an operating microscope, as previously described [26], and 5 min of reperfusion.

Mice were randomly divided into the following five groups (Fig. 1): 1) Sham, in which the ligature was placed under the LCA without occlusion; 2) IR, in which mice were subjected to 45 min of coronary artery occlusion followed by $2 \mathrm{~h}$ of reperfusion; 3) RIPostC, in which mice received a left hind limb intervention with 3 cycles of $5 \mathrm{~min}$ of reperfusion followed by $5 \mathrm{~min}$ of ischaemia immediately at the onset of the coronary reperfusion period; 4) RIPostC+AG490, in which mice received the JAK/STAT3 inhibitor AG490 (20 mg/kg i.p., dissolved in 5\% dimethyl sulfoxide (DMSO), Sigma-Aldrich, Germany) 10 min before the start of reperfusion and underwent a left hind limb intervention with 3 cycles of 5 min of reperfusion followed by 5 min of ischaemia at the onset of coronary reperfusion; and 5) RIPostC+DMSO, in which mice received the same volume of DMSO 10 min before the start of reperfusion and underwent a left hind limb intervention with 3 cycles of $5 \mathrm{~min}$ of reperfusion followed by 5 min of ischaemia at the onset of coronary reperfusion.

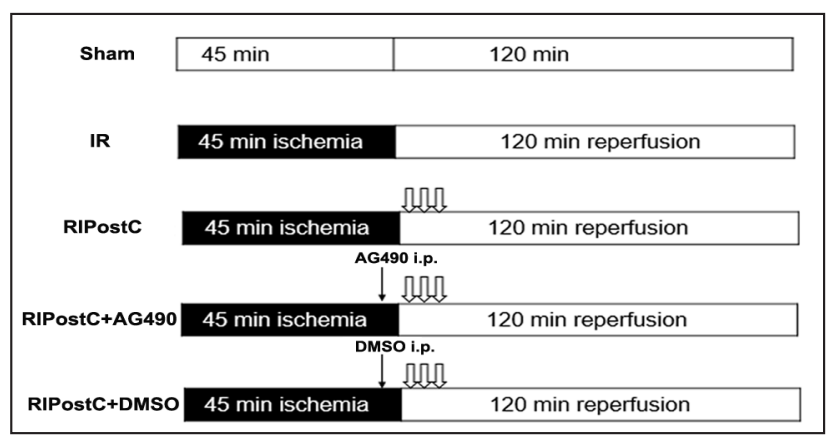

Fig. 1. Experimental groups and protocol. 


\section{Cellular Physiology Cell Physiol Biochem 2017;43:1140-1151 \begin{tabular}{l|l|l} 
DOI: 10.1159/000481755 & $\begin{array}{l}\text { O 2017 The Author(s). Published by S. Karger AG, Basel } \\
\text { www.karger.com/cpb }\end{array}$
\end{tabular} \\ Gao et al.: Nrf2-Antioxidant Signalling in Remote Limb Ischaemic Postconditioning Cardioprotection}

\section{Determination of risk and infarct sizes}

At the end of perfusion, the LCA was reoccluded at the same site, and $0.3 \mathrm{ml}$ of $2 \%$ Evans blue dye was injected into the abdominal vein to identify the area at risk (AAR), which would remain unstained in contrast to the blue, non-ischaemic part of the myocardium. The heart was then frozen at $-20^{\circ} \mathrm{C}$ for 20 min and cut transversely into four 1-mm-thick sections. After being incubated in 2\% 2, 3, 5-triphenyltetrazolium chloride (TTC, Sigma-Aldrich, Germany) solution for $20 \mathrm{~min}$ at $37^{\circ} \mathrm{C}$, the slices were fixed in $4 \%$ paraformaldehyde solution overnight to identify viable myocardium, which stained red while the necrotic (infarcted) tissue remained pale. The AAR is expressed as a percentage of the total weight, and the infarct size (IS) is expressed as a percentage of the weight of the AAR. The extent of the area of necrosis was quantified by computerized planimetry (ImageJ 1.4).

\section{Measurement of plasma creatine kinase-MB (CK-MB) and lactate dehydrogenase ( $L D H)$ levels}

After $2 \mathrm{~h}$ of reperfusion, blood samples were collected for measurement of CK-MB by an enzyme immunoassay using a commercial kit (USCN Life Science Inc., China) and LDH by a colourimetric method using a commercially available assay kit (Jiancheng Bioengineering Institute, China), according to the manufacturer's instructions.

\section{Detection of Myocardial Apoptosis}

Myocardial apoptosis was assessed through a TUNEL assay. Samples of paraformaldehyde-fixed myocardial tissue embedded in paraffin were measured with an in situ cell detection kit (Roche Diagnostics, Germany) to identify the apoptotic cells according to the manufacturer's protocol, using a fluorescence microscope. TUNEL-positive cells were counted under a high-power field (magnification, $\times 400$ ). A total of three fields per heart were analysed, and the mean \pm SD was determined.

Measurement of malondialdehyde (MDA) and superoxide dismutase (SOD) levels

After reperfusion, blood samples were collected for measurement of MDA by the thiobarbituric acid method using an assay kit (Jiancheng Bioengineering Institute, China) and of SOD by the hydroxylamine method using an assay kit (Jiancheng Bioengineering Institute, China).

\section{Detection of superoxide generation}

Superoxide generation was assessed by fluorescent-labelled dihydroethidium (Keygen Biotech Co., China) staining. Dihydroethidium is cell permeable and reacts with superoxide to form ethidium, which, in turn, intercalates with DNA and produces nuclear fluorescence. The procedure was conducted in accordance with the assay kit protocol.

\section{Western blot analysis}

Cytoplasmic and nuclear proteins were extracted from heart tissue using a Protein Extraction Kit (Keygen Biotech CO., China) containing protease inhibitor and phosphatase inhibitor cocktails (Sigma Aldrich, Germany), according to the manufacturer's protocols. Protein concentration was measured by the bicinchoninic acid method (Sigma Aldrich, Germany). Before the immunoblotting, aliquots of lysate were mixed with $5 \times$ loading buffer containing 2-mercaptoethanol and maintained at $100^{\circ} \mathrm{C}$ for $10 \mathrm{~min}$. Equal amounts of protein $(50 \mu \mathrm{g})$ were loaded and electrophoresed on $10 \%$ SDS-polyacrylamide gels and were subsequently transferred to PVDF membranes. The membranes were blocked for $1 \mathrm{~h}$ at room temperature with TBST containing 5\% (w/v) non-fat milk. Membrane strips were then incubated at $4{ }^{\circ} \mathrm{C}$ overnight with primary antibodies at the following dilutions: phospho-Akt (Ser473), Akt and cleaved caspase 3 (Cell Signaling Technology, USA) at 1:1000; phospho-STAT3 (Tyr705), STAT3, phospho-eNOS (Ser1177), eNOS and Nrf2 (Abcam, UK) at 1:500; and HO-1 (ABclonal, China) at 1:1000. Following extensive washing, primary antibody binding was detected with a secondary antibody (Cell Signaling Technology, USA) at 1:5000. Protein bands were detected by a standard ECL method, and images were measured by a densitometer with analysis software (ImageJ 1.4).

\section{Statistical analysis}

All values are reported as the mean \pm SD. Data analysis was performed with a personal computer statistical software package (GraphPad Prism, San Diego, CA). One-way or two-way ANOVA was used to 
Gao et al.: Nrf2-Antioxidant Signalling in Remote Limb Ischaemic Postconditioning Cardioprotection

determine significant differences within groups and between groups, respectively, followed by the Newman-Keuls method for multiple comparisons of group means. Differences were considered statistically significant when their P values were $<0.05$.

\section{Results}

\section{Haemodynamic parameters}

The mean arterial blood pressure (MAP) and heart rate were recorded throughout the experimental protocols in all groups. Haemodynamic data are shown in Table 1. There was no significant difference in heart rate across groups, either at baseline or throughout the experiments $(P>0.05)$. The MAP of all groups except the Sham group was significantly decreased during myocardial ischaemia and reperfusion compared with the corresponding baseline measurements $(\mathrm{P}<0.01)$. However, the MAP was remarkably elevated in the RIPostC and RIPostC+DMSO groups compared with the IR group during the $2 \mathrm{~h}$ of reperfusion $(P<0.05)$. Interestingly, AG490 but not DMSO blocked the effect of RIPostC in elevating MAP during the $2 \mathrm{~h}$ of reperfusion $(\mathrm{P}<0.05)$.

\section{Infarct size and area at risk}

As shown in Fig. 2, the AAR did not differ significantly among the five experimental groups $(\mathrm{P}>$ 0.05). RIPostC significantly reduced the infarct size caused by IR injury (RIPostC group, $22.9 \% \pm 3.3 \%$, vs IR group, $40.9 \% \pm 6.2 \% ; \mathrm{P}<0.01$ ). The infarct size-limiting effect of RIPostC was nearly abolished by AG490 (RIPostC+AG490 group, $38.8 \pm 3.7 \%$, vs RIPostC group, 22.9\% $\pm 3.3 \%$; $\mathrm{P}<0.01$ ). DMSO did not affect the cardioprotective effect of RIPostC in reducing infarct size in mice (RIPostC+DMSO group, $22.7 \% \pm 3.9 \%$, vs RIPostC group, $22.9 \% \pm 3.3 \% ; \mathrm{P}>0.05$ ).

\section{Cardiac enzyme release}

Leakage of CK-MB isoenzyme and $\mathrm{LDH}$ from the myocardial tissues to the blood is an indicator of acute myocardial infarction. As shown in Fig. 3, the release of CK-MB and LDH was greatly increased after myocardial IR injury in mice (IR vs Sham group, $\mathrm{P}<0.01$ ).
Table 1. Hemodynamic parameters. All values are expressed mean arterial pressure (MAP) were measured at baseline and during ischemia/reperfusion. ${ }^{* *} \mathrm{P}<0.01$ VS their corresponding Baseline; \# $\mathrm{P}<0.05$ VS their corresponding IR groups; ${ }^{\&} \mathrm{P}<0.05$ VS their corresponding RIPostC groups

\begin{tabular}{lcllll}
\hline Parameters & Sham & IR & RIPostC & RIPostC+AG490 & RIPostC+DMSO \\
\hline $\begin{array}{l}\text { Heart rate (beats/min) } \\
\text { Baseline }\end{array}$ & $362 \pm 4$ & $369 \pm 15$ & $379 \pm 16$ & $366 \pm 9$ & $362 \pm 4$ \\
Ischemia 15 min & $369 \pm 9$ & $372 \pm 18$ & $380 \pm 22$ & $369 \pm 9$ & $361 \pm 12$ \\
Reperfusion 2 h & $369 \pm 13$ & $370 \pm 13$ & $376 \pm 12$ & $367 \pm 16$ & $364 \pm 8$ \\
Mean arterial blood pressure ( $\mathrm{mmHg}$ ) & & & & \\
Baseline & $84 \pm 9$ & $81 \pm 6$ & $83 \pm 5$ & $78 \pm 3$ & $83 \pm 4$ \\
Ischemia 15 min & $80 \pm 9$ & $60 \pm 4^{* *}$ & $62 \pm 6^{* *}$ & $62 \pm 6^{* *}$ & $61 \pm 6^{* *}$ \\
Reperfusion 2 h & $85 \pm 5$ & $57 \pm 5^{* *}$ & $66 \pm 4^{* * * *}$ & $57 \pm 4^{* * *}$ & $67 \pm 6^{* * *}$ \\
\hline
\end{tabular}

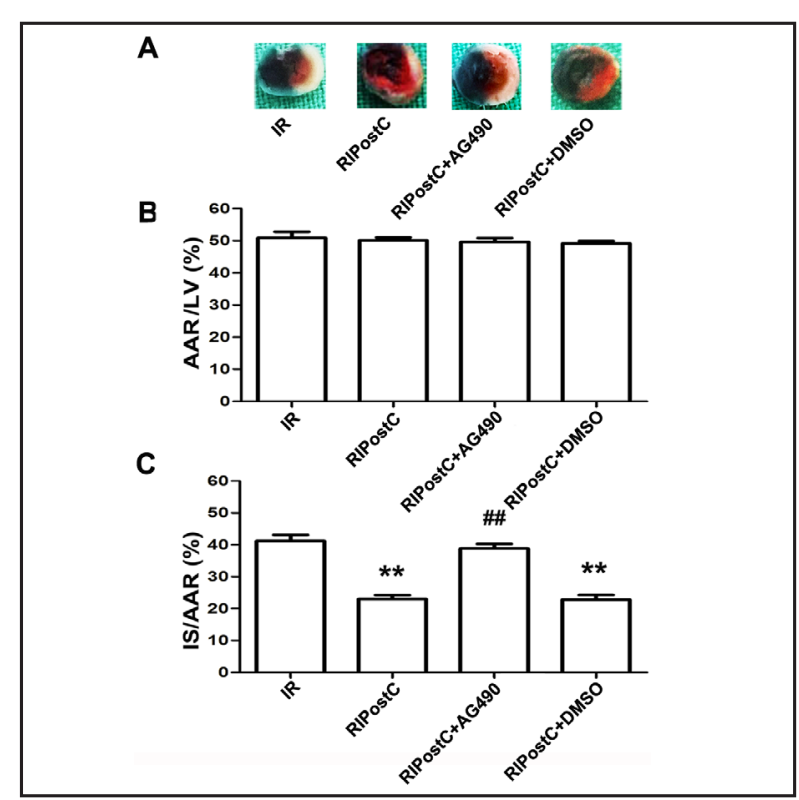

Fig. 2. Myocardial ischemia reperfusion injury expressed as percentage infarct size. (A) Representative images of nonischemic zones (blue) and infracted tissue (white) within the AAR (absence of blue dye). (B) In all groups, AAR did not differ significantly. (C) The infarct size was reduced in RIPostC group. Presented values are mean \pm SD. $n=7$ per group. ${ }^{* *} \mathrm{P}<0.01$ compared with IR group; \#\# $\mathrm{P}<0.01$ compared with RIPostC group. as mean \pm SD. $n=5$ for per group. Heart rate (HR) and 


\section{Cellular Physiology \begin{tabular}{ll|l} 
and Biochemistry & $\begin{array}{l}\text { DOI: 10.1159/000481755 } \\
\text { Published online: October 05, } 2017\end{array}$ & $\begin{array}{l}\text { (c) } 2017 \text { The Author(s). Published by S. Karger AG, Basel } \\
\text { www.karger.com/cpb }\end{array}$ \\
\hline
\end{tabular} \\ Gao et al.: Nrf2-Antioxidant Signalling in Remote Limb Ischaemic Postconditioning Cardioprotection}

Fig. 3. Myocardial ischemia reperfusion injury expressed as plasma CK-MB and LDH secretion. (A) The level of CK-MB secretion was decreased in RIPostC. (B) The level of LDH secretion was decreased in RIPostC group. Presented values are mean \pm SD. $\mathrm{n}=5$ per group. ${ }^{* *} \mathrm{P}<0.01$ compared with IR group; \#\# $\mathrm{P}<0.01$ compared with RIPostC group.

The RIPostC group had a significantly lesser release of CK-MB and LDH than the IR group $(\mathrm{P}<0.01)$. AG490 abolished the effect of RIPostC in decreasing plasma CK-MB and LDH secretion in mice (RIPostC+AG490 vs RIPostC group, $\mathrm{P}<0.01$; RIPostC+AG490 vs IR group, $P>0.05)$. There was no significant difference in CK-MB or LDH release between the RIPostC and RIPostC+DMSO groups $(\mathrm{P}>0.05)$.

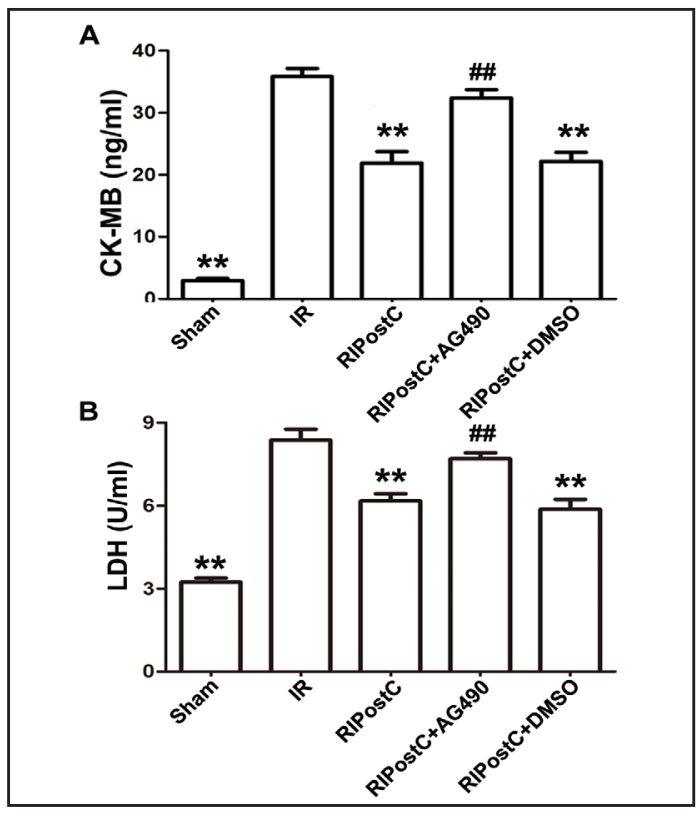

Analysis of apoptotic cells and cleaved caspase 3

Apoptosis is characterized by chromatin condensation and fragmentation, cell shrinkage, and plasma membrane budding with release of apoptotic bodies that are phagocytized [27]. As shown in Fig. 4 A and B, quantification of TUNEL-positive myocyte nuclei revealed that the IR group contained significantly more apoptotic cells than the RIPostC group or the RIPostC+DMSO group (IR group, $26.2 \% \pm 3.1 \%$, vs RIPostC group, $13.4 \% \pm 3.1 \%$, or RIPostC+DMSO group, $13.3 \% \pm 3.0 \%$, respectively; $\mathrm{P}<0.01$ ). However, the anti-apoptotic effect of RIPostC was almost completely abolished by AG490 (RIPostC+AG490 vs RIPostC group, $\mathrm{P}<0.05$; RIPostC+AG490 vs IR group, $\mathrm{P}>0.05$ ).

Cleaved (active) caspase 3 is known to play a central role in cellular apoptosis. As shown in Fig. $4 \mathrm{C}$ and D, both the RIPostC and RIPostC+DMSO groups produced remarkably less cleaved caspase 3 than the IR group ( $P<0.01)$, while pretreatment with AG490 significantly abrogated the effect of RIPostC $(\mathrm{P}<0.01)$.

\section{Analysis of STAT3, Akt and eNOS phosphorylation}

As shown in Fig. 5, the phosphorylation of STAT3, Akt and eNOS was significantly increased after myocardial IR insult compared with the Sham group $(\mathrm{P}<0.01)$. In addition, RIPostC further increased the phosphorylation of STAT3, Akt and eNOS compared with the levels in the IR group ( $\mathrm{P}<0.01)$. However, AG490 remarkably suppressed the phosphorylation of STAT3, Akt and eNOS induced by RIPostC $(\mathrm{P}<0.05)$.

\section{Analysis of oxidative stress}

As shown in Fig. $6 \mathrm{~A}$, the immunofluorescence images revealed that myocardial superoxide anion generation (number of dihydroethidium-labelled nuclei) was significantly increased in the IR group compared with the Sham group $(\mathrm{P}<0.01)$. RIPostC markedly decreased superoxide anion generation in mice, and the effect of RIPostC was blocked by AG490. The level of the lipid oxidation marker MDA in the IR group was higher than that in the Sham group, and the level was remarkably decreased by RIPostC (Fig. 6 B, P < 0.01). After pretreatment with AG490, the level of MDA in the RIPostC+AG490 group was elevated compared with the RIPostC group ( $\mathrm{P}<0.01)$. In addition, as shown in Fig. $6 \mathrm{C}$, the antioxidant enzyme SOD was partly consumed in all groups after IR and restored by RIPostC. However, the effect of RIPostC in restoring SOD was abolished by AG490 (RIPostC+AG490 vs RIPostC group, $\mathrm{P}<0.05$ ). 
Fig. 4. Myocardial apoptosis detected by TUNEL staining and the protein expression of cleaved caspase 3 (CC3). (A) TUNEL staining. TUNEL staining (green) indicates apoptotic cells, DAPI counterstaining (blue) indicates total nuclei (magnification, $\times 400$ ). (B) Bar diagram showing quantitative data of TUNEL. (C) The protein expression of CC3 was detected by western blot. (D) Bar graph showing the quantification of the immunoreactive band obtained as above. Presented values are mean \pm SD. $\mathrm{n}=5$ per group. ${ }^{* *} \mathrm{P}<0.01$ compared with IR group; \# $\mathrm{P}<0.05$ compared with RIPostC group; \#\# $\mathrm{P}<0.01$ compared with RIPostC group.

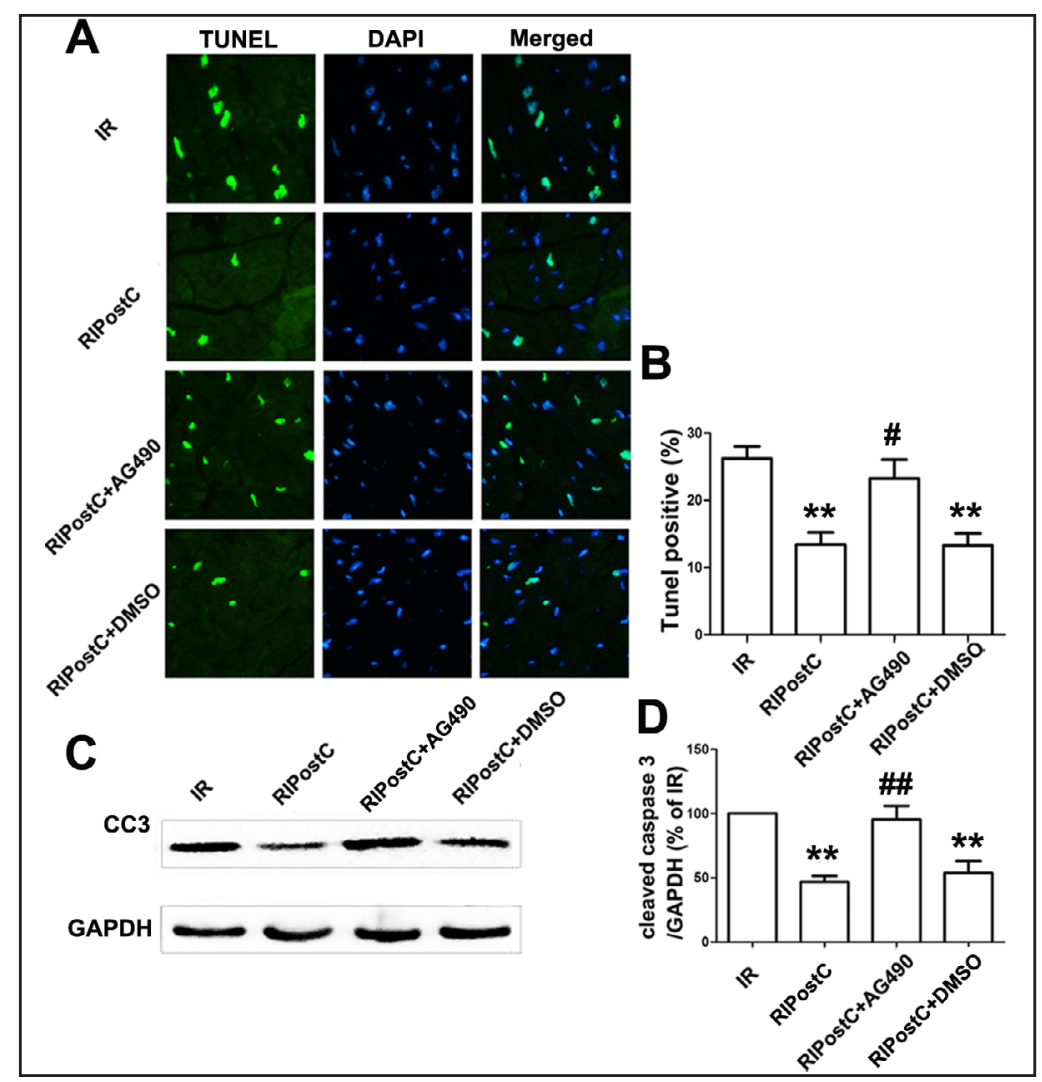

Fig. 5. Cardiac protein expression of STAT3, p-STAT3 (Tyr705), Akt, eNOS, pAkt (Ser473) and p-eNOS (Ser1177) after myocardial IR detected by western blotting. (A) Representative Western blots. (B-D) Bar graph showing the quantification of the immunoreactive band obtained as above. Presented values are mean \pm SD. $n=5$ per group. ${ }^{* *} \mathrm{P}<0.01$ compared with IR group; \# $\mathrm{P}<0.05$ compared with RIPostC group; ${ }^{\# \# ~} \mathrm{P}<0.01$ compared with RIPostC group.

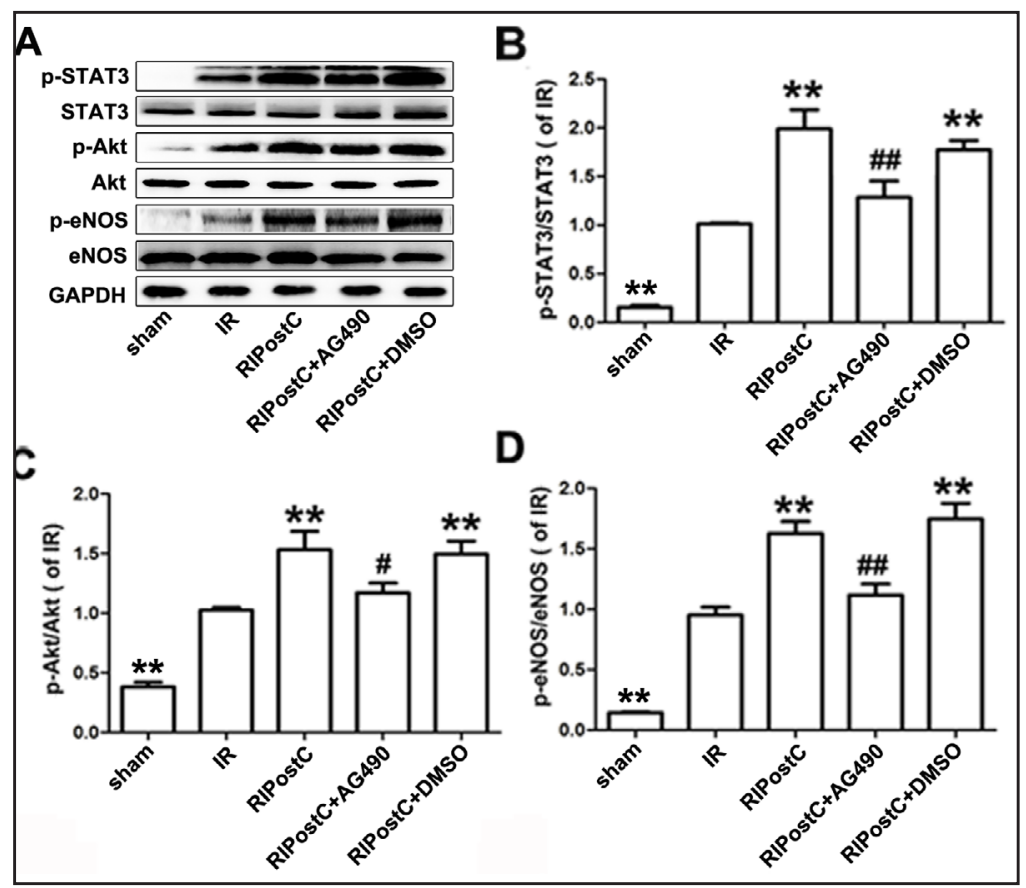

Analysis of Nrf2 and $\mathrm{HO}-1$

As shown in Fig. $6 \mathrm{D}$, the nuclear translocation of Nrf2 was significantly induced by IR insult in mice and further increased by RIPostC (Sham vs IR, P < 0.01; RIPostC vs IR, $\mathrm{P}<0.05)$. However, the nuclear translocation of Nrf2 was significantly blunted by AG490 (RIPostC+AG490 vs RIPostC, $\mathrm{P}<0.05)$. As shown in Fig. 6 E, RIPostC significantly increased 


\section{$\begin{array}{ll}\text { Cellular Physiology } & \text { Cell Physiol Biochem 2017;43:1140-1151 } \\ \text { DOI: 10.1159/000481755 } & 0 \text { 2017 The Author(s). Published by S. Karger AG, Basel }\end{array}$

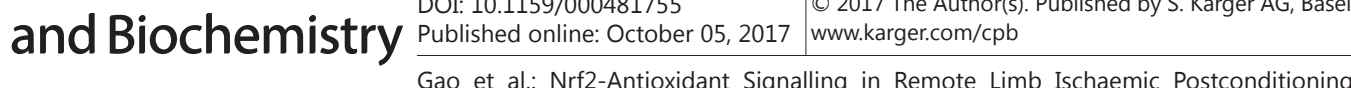

Fig. 6. Oxidant products and anti-oxidant enzyme measured after myocardial IR. Cardiac protein expression of Nrf2 and HO-1 after myocardial IR detected by western blotting. (A) Photographs of dihydroethidium (DHE) staining detected by immunofluorescence. (B) Detection of malondialdehyde (MDA) levels assessed by a kit. (C) Detection of superoxide dismutase (SOD) activity assessed by a kit. (D-E) Representative Western blots. Bar graph showing the quantification of the immunoreactive band

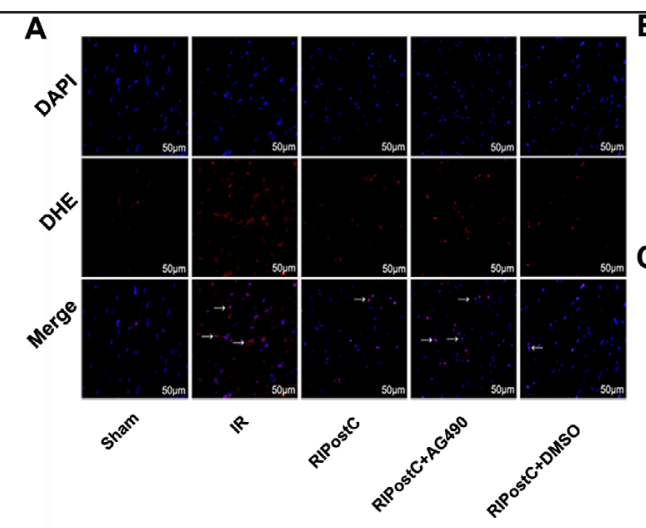

D

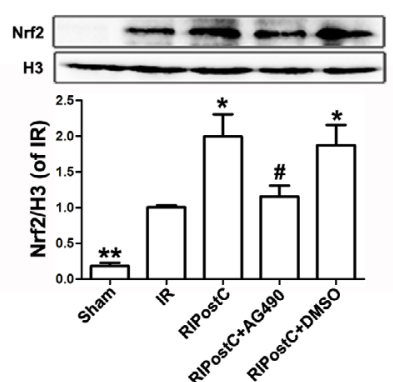

E

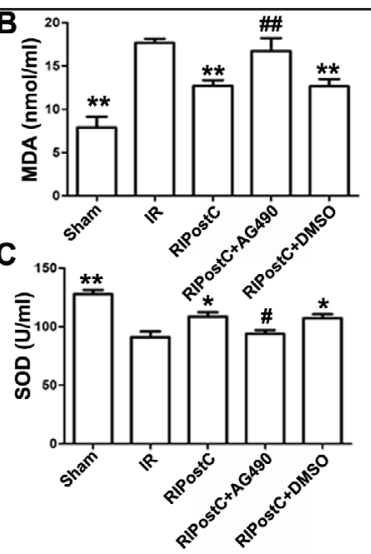

E
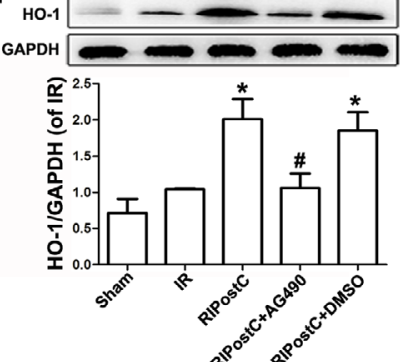
obtained as above. Presented values are mean \pm SD. $n=5$ per group. ${ }^{*} \mathrm{P}<0.05$ compared with IR group; ${ }^{* *} \mathrm{P}<0.01$ compared with IR group; \# $\mathrm{P}<0.05$ compared with RIPostC group; ${ }^{\# \# ~} \mathrm{P}<0.01$ compared with RIPostC group.

myocardial HO-1 expression in mice, and the effect of RIPostC was markedly inhibited by AG490 $(\mathrm{P}<0.05)$.

\section{Discussion}

There were several novel findings in our current study. First, we demonstrated that RIPostC attenuated apoptosis and protected against myocardial IR injury in mice. Second, the cardioprotective effects induced by RIPostC were associated with increased phosphorylation of myocardial STAT3, Akt, and eNOS. Third, RIPostC increased the nuclear translocation of Nrf2 and the expression of HO-1, protecting against oxidative stress induced by myocardial IR injury. More interestingly, the JAK/STAT3 inhibitor AG490 remarkably blocked the cardioprotective effect of RIPostC due to the inhibition of myocardial STAT3, Akt and eNOS phosphorylation as well as antioxidant elements.

Myocardial IR injury is a major cause of morbidity and mortality after cardiac operations and myocardial infarctions. RIPostC is an experimental intervention that has the potential to contribute to myocardial protection following ischaemic insult $[11,28]$. Studies have shown that the infarct size plays an important role in the onset and extent of irreversible cellular injury $[29,30]$. In our study, we found that RIPostC attenuated the myocardial infarct size after 45 min of ischaemia and $2 \mathrm{~h}$ of reperfusion in mice, which suggested that RIPostC significantly decreased the myocardial injury after IR insult. CK-MB release of more than five to eight times the upper limit of the reference range is associated with an increased risk of death [31]. In the RIPostC group, CK-MB and LDH release were powerfully reduced, which indicated that the risk of myocardial death might be decreased. Cleaved caspase 3 , which is the activated fragment of caspase 3 , can cleave the downstream death substrates and amplify the upstream death cascade, which causes apoptosis of cells [32]. In our study, Western blot analysis showed that the RIPostC group had a lower level of cleaved caspase 3 than the IR 
group and that RIPostC could inhibit the activation of pro-apoptotic substrates. In addition, a TUNEL assay showed that the RIPostC group had significantly fewer apoptotic cells than the IR group. These results demonstrated that RIPostC significantly increased the number of surviving cardiomyocytes and decreased myocardial apoptosis in the ischaemic penumbra following myocardial IR insult. It is important to note that although cardiomyocytes are the predominant cell type in the heart, TUNEL staining is not cardiomyocyte specific; thus, other cell types such as endothelial cells may have been stained in our TUNEL study, and we could not rule out the possibility that RIPostC may also effective in attenuating cell apoptosis in types of cells other than cardiomyocytes. Nevertheless, our results suggested that RIPostC provided powerful cardioprotection against IR injury in mice.

STAT3 is an important regulator of cardiac function and plays key roles in cardioprotection through activation of cellular survival pathways [33]. STAT3 is activated by numerous ligand receptors, such as the IL-6-glycoprotein (gp) 130 receptor system [34], the leptin receptor [35], the EPO receptor [36], and the AngII type 1 receptor [37]. The IL-6-gp130 receptor system, which activates phosphorylation of STAT3 at tyrosine 705 (Tyr705) and serine 727 (Ser727), is currently considered the major regulator of STAT3. Phosphorylation of STAT3 at Tyr705 is mediated by JAK at the intracytoplasmic domain of the gp130 receptor [38]. Importantly, the JAK-STAT3 pathway has been shown to be essential to the cardioprotection induced by ischaemic postconditioning [39]. In agreement with our previous study [18], we found that the expression of p-STAT3 Tyr705 was increased following myocardial IR injury. Furthermore, the RIPostC group showed more pronounced increase in p-STAT3 Tyr705 expression than the IR group, and that elevation may contribute to the decreased number of apoptotic cardiomyocytes and the attenuation of myocardial infarct size. Although the downstream protective targets activated by STAT3 have not been systematically investigated, some research has reported that phosphorylation of STAT3 can activate downstream protective substrates, including Akt, eNOS, and GSK3 $\beta[18,40]$. STAT3, Akt and eNOS have been shown to inhibit opening of the MPTP during reperfusion [41-43], which can maintain mitochondrial membrane integrity and inhibit the activation of the mitochondrial apoptosis pathway. In our study, we indeed observed that RIPostC increased Akt and eNOS Ser1177 phosphorylation and that pretreatment with the JAK/STAT3 inhibitor AG490 attenuated the phosphorylation of STAT3 as well as the phosphorylation of Akt and eNOS. Therefore, these results suggest that a JAK/STAT3-mediated Akt and eNOS signalling pathway may play a compelling role in the cardioprotection induced by RIPostC.

$\mathrm{Nrf2}$ is a master redox regulator of the antioxidant defence system, including HO-1, quinone oxidoreductase-1 (NQO1), and SOD. Nrf2-antioxidant signalling plays a significant role in protecting cells against oxidative stress [44]. In our study, RIPostC promoted the translocation of Nrf2 into the nucleus. Studies have shown that RIPostC in one limb alleviated reperfusion injury after focal cerebral ischaemia through ROS-mediated inhibition of endogenous $\delta$-PKC activation and the related signalling cascade in an in vivo rat model of focal cerebral ischaemia [45]. Hence, we speculated that RIPostC could stimulate small-scale production of ROS to activate the Nrf2 pathway. However, the antioxidative effect of Nrf2, which is a transcription factor, depends on its downstream antioxidants, such as HO-1 and SOD. The antioxidants HO- 1 and SOD play an important role in cardiovascular protection [46, 47]. In the present study, RIPostC markedly elevated the expression of HO-1 and SOD and subsequently reduced oxidative stress after myocardial IR. Interestingly, the STAT3 inhibitor AG490 blocked the nuclear translocation of Nrf2 and the expression of HO-1. These results demonstrated that RIPostC-induced myocardial STAT3 activation could regulate the nuclear translocation of Nrf2 to promote a series of antioxidant genes. Indeed, studies using the NRF2-ome website have indicated the possibility of a connection between Nrf2 and the JAK/ STAT pathway through STAT1 and STAT3 [48]. Moreover, many studies have demonstrated that the PI3K/Akt signalling pathway can regulate the nuclear translocation of Nrf2 $[49,50]$. Therefore, these investigations suggest that RIPostC increases the nuclear translocation of Nrf2 and the expression of HO-1 against excessive oxidative stress after myocardial IR injury and that this response is mediated by the STAT3/Akt/eNOS signalling pathway.

\section{KARGER}




\section{Cellular Physiology Cell Physiol Biochem 2017;43:1140-1151 \begin{tabular}{ll|l} 
DOI: 10.1159/000481755 & $\begin{array}{l}\text { O } 2017 \text { The Author(s). Published by S. Karger AG, Basel } \\
\text { www.karger.com/cpb }\end{array}$ \\
\hline and Biochemistry
\end{tabular} \\ Gao et al.: Nrf2-Antioxidant Signalling in Remote Limb Ischaemic Postconditioning Cardioprotection}

This study has some limitations. First, we have found that RIPostC can protect against myocardial IR injury in the acute stage, but it is still unknown whether RIPostC can provide cardioprotective effects in the chronic stage and improve heart function. Second, although cardiomyocytes are the predominant cell type in the heart, TUNEL staining is not cardiomyocyte specific. Thus, other cell types such as endothelial cells may have been stained in our TUNEL study, and we could not rule out the possibility that RIPostC may also be effective in attenuating cell apoptosis in types of cells other than cardiomyocytes. Nevertheless, these results suggested that RIPostC provided powerful cardioprotection against IR injury in mice. Further research is needed for a better understanding of the specific mechanism by which RIPostC acts on the STAT3/Nrf2/HO-1 pathway.

\section{Conclusion}

RIPostC attenuated apoptosis and protected against myocardial IR injury, possibly through the activation of JAK/STAT3-mediated Nrf2-antioxidant signalling pathway in mice. Our results provided insight into the effects of mechanisms of RIPostC, which may lead to the development of effective therapeutic regimens to combat myocardial IR insult.

\section{Acknowledgements}

This study was supported by grants from the National Natural Science Foundation of China (No. 81200609 and No. 81370112).

\section{Disclosure Statement}

The authors declare that there is no conflict of interest regarding the publication of this paper.

\section{References}

1 Thielmann M, Kottenberg E, Kleinbongard P, Wendt D, Gedik N, Pasa S, Price V, Tsagakis K, Neuhäuser M, Peters J, Jakob H, Heusch G: Cardioprotective and prognostic effects of remote ischaemic preconditioning in patients undergoing coronary artery bypass surgery: a single-centre randomised, double-blind, controlled trial. Lancet 2013;382:597-604.

2 Laurikka J, Wu ZK, Iisalo P, Kaukinen L, Honkonen EL, Kaukinen S, Tarkka MR: Regional ischemic preconditioning enhances myocardial performance in off-pump coronary artery bypass grafting. Chest 2002;121:1183-1189.

-3 Hahn JY, Song YB, Kim EK, Yu CW, Bae JW, Chung WY, Choi SH, Choi JH, Bae JH, An KJ, Park JS, Oh JH, Kim SW, Hwang JY, Ryu JK, Park HS, Lim DS, Gwon HC: Ischemic postconditioning during primary percutaneous coronary intervention: the effects of postconditioning on myocardial reperfusion in patients with STsegment elevation myocardial infarction (POST) randomized trial. Circulation 2013;128:1889-1896.

-4 Crimi G, Pica S, Raineri C, Bramucci E, De Ferrari GM, Klersy C, Ferlini M, Marinoni B, Repetto A, Romeo M, Rosti V, Massa M, Raisaro A, Leonardi S, Rubartelli P, Oltrona Visconti L, Ferrario M: Remote ischemic post-conditioning of the lower limb during primary percutaneous coronary intervention safely reduces enzymatic infarct size in anterior myocardial infarction: a randomized controlled trial. JACC Cardiovasc Interv 2013;6:1055-1063.

5 Candilio L, Malik A, Ariti C, Barnard M, Di Salvo C, Lawrence D, Hayward M, Yap J, Roberts N, Sheikh A, Kolvekar S, Hausenloy DJ, Yellon DM: Effect of remote ischaemic preconditioning on clinical outcomes in patients undergoing cardiac bypass surgery: a randomised controlled clinical trial. Heart 2015;101:185192.

6 Zhao ZQ, Corvera JS, Halkos ME, Kerendi F, Wang NP, Guyton RA, Vinten-Johansen J: Inhibition of myocardial injury by ischemic postconditioning during reperfusion: comparison with ischemic preconditioning. Am J Physiol Heart Circ Physiol 2003;285:H579-H588.

\section{KARGER}




\section{Cellular Physiology Cell Physiol Biochem 2017;43:1140-1151

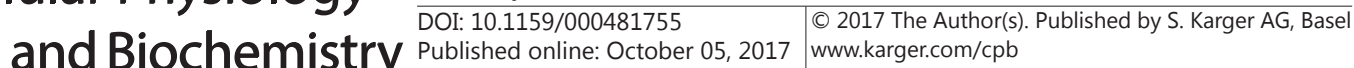

Gao et al.: Nrf2-Antioxidant Signalling in Remote Limb Ischaemic Postconditioning Cardioprotection

7 Jiménez-Navarro MF, Carrasco-Chinchilla F, Muñoz-García AJ, Domínguez-Franco A, Caballero-Borrego J, Alonso-Briales JH, Hernández-García JM, de Teresa-Galván E: Remote Ischemic Postconditioning: Does It Protect against Ischemic Damage in Percutaneous Coronary Revascularization? Justification and Design of a Randomized Placebo-Controlled Clinical Trial. Cardiology 2011;119:164-169.

8 Zhong H, Gao Z, Chen M, Zhao J, Wang F, Li L, Dong H, Liu L, Wang Q, Xiong L: Cardioprotective effect of remote ischemic postconditioning on children undergoing cardiac surgery: a randomized controlled trial. Paediatr Anaesth 2013;23:726-733.

9 Heusch G, Rassaf T: Time to Give Up on Cardioprotection? A Critical Appraisal of Clinical Studies on Ischemic Pre-, Post-, and Remote Conditioning. Circ Res 2016;119:676-695.

10 Hausenloy DJ, Yellon DM: Ischaemic conditioning and reperfusion injury. Nat Rev Cardiol 2016;13:193-209.

11 Han Z, Cao J, Song D, Tian L, Chen K, Wang Y, Gao L, Yin Z, Fan Y, Wang C: Autophagy is involved in the cardioprotection effect of remote limb ischemic postconditioning on myocardial ischemia/reperfusion injury in normal mice, but not diabetic mice. Plos One 2014;9:e86838.

12 Gao Y, Song J, Chen H, Cao C, Lee C: TRPV1 activation is involved in the cardioprotection of remote limb ischemic postconditioning in ischemia-reperfusion injury rats. Biochem Bioph Res Co 2015;463:10341039.

13 Gedik N, Thielmann M, Kottenberg E, Peters J, Jakob H, Heusch G, Kleinbongard P: No Evidence for Activated Autophagy in Left Ventricular Myocardium at Early Reperfusion with Protection by Remote Ischemic Preconditioning in Patients Undergoing Coronary Artery Bypass Grafting. Plos One 2014;9:e96567.

14 Lacerda L, Somers S, Opie LH, Lecour S: Ischaemic postconditioning protects against reperfusion injury via the SAFE pathway. Cardiovasc Res 2009;84:201-208.

15 Heusch G, Musiolik J, Gedik N, Skyschally A: Mitochondrial STAT3 Activation and Cardioprotection by Ischemic Postconditioning in Pigs With Regional Myocardial Ischemia/Reperfusion. Circ Res 2011;109:1302-1308.

-16 Boengler K, Buechert A, Heinen Y, Roeskes C, Hilfiker-Kleiner D, Heusch G, Schulz R: Cardioprotection by Ischemic Postconditioning Is Lost in Aged and STAT3-Deficient Mice. Circ Res 2008;102:131-135.

$\checkmark 17$ O Sullivan KE, Breen EP, Gallagher HC, Buggy DJ, Hurley JP: Understanding STAT3 signaling in cardiac ischemia. Basic Res Cardiol 2016;111:27.

18 Wang T, Mao X, Li H, Qiao S, Xu A, Wang J, Lei S, Liu Z, Ng KF, Wong GT, Vanhoutte PM, Irwin MG, Xia Z: $\mathrm{N}$-Acetylcysteine and allopurinol up-regulated the Jak/STAT3 and PI3K/Akt pathways via adiponectin and attenuated myocardial postischemic injury in diabetes. Free Radical Bio Med 2013;63:291-303.

19 Miura T, Tanno M: The mPTP and its regulatory proteins: final common targets of signalling pathways for protection against necrosis. Cardiovasc Res 2012;94:181-189.

20 Ibanez B, Heusch G, Ovize M, Van de Werf F: Evolving Therapies for Myocardial Ischemia/Reperfusion Injury. J Am Coll Cardiol 2015;65:1454-1471.

-21 Heusch G: Molecular basis of cardioprotection: signal transduction in ischemic pre-, post-, and remote conditioning. Circ Res 2015;116:674-699.

-22 Ichihara S: The pathological roles of environmental and redox stresses in cardiovascular diseases. Environ Health Prev Med 2013;18:177-184.

-23 McMahon M, Itoh K, Yamamoto M, Hayes JD: Keap1-dependent proteasomal degradation of transcription factor Nrf2 contributes to the negative regulation of antioxidant response element-driven gene expression. J Biol Chem 2003;278:21592-21600.

24 Ishii T, Mann GE: Redox status in mammalian cells and stem cells during culture in vitro: critical roles of $\mathrm{Nrf} 2$ and cystine transporter activity in the maintenance of redox balance. Redox Biol 2014;2:786-794.

25 Howden R: Nrf2 and cardiovascular defense. Oxid Med Cell Longev 2013;2013:104308.

-26 Wang F, Birch SE, He R, Tawadros P, Szaszi K, Kapus A, Rotstein OD: Remote Ischemic Preconditioning by Hindlimb Occlusion Prevents Liver Ischemic/Reperfusion Injury. Ann Surg 2010;251:292-299.

27 Kerr JF, Wyllie AH, Currie AR: Apoptosis: a basic biological phenomenon with wide-ranging implications in tissue kinetics. Br J Cancer 1972;26:239-257.

-28 Jiang Q, Song P, Wang E, Li J, Hu S, Zhang H: Remote ischemic postconditioning enhances cell retention in the myocardium after intravenous administration of bone marrow mesenchymal stromal cells. J Mol Cell Cardiol 2013;56:1-7. 


\section{Cellular Physiology Cell Physiol Biochem 2017;43:1140-1151

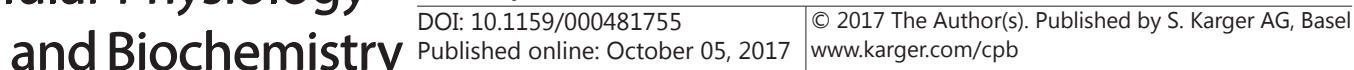

Gao et al.: Nrf2-Antioxidant Signalling in Remote Limb Ischaemic Postconditioning Cardioprotection

-29 Reimer KA, Jennings RB: The "wavefront phenomenon" of myocardial ischemic cell death. II. Transmural progression of necrosis within the framework of ischemic bed size (myocardium at risk) and collateral flow. Lab Invest 1979;40:633-644.

30 Ytrehus K, Liu Y, Tsuchida A, Miura T, Liu GS, Yang XM, Herbert D, Cohen MV, Downey JM: Rat and rabbit heart infarction: effects of anesthesia, perfusate, risk zone, and method of infarct sizing. Am J Physiol 1994;267:H2383-H2390.

-31 Petaja L, Salmenpera M, Pulkki K, Pettila V: Biochemical injury markers and mortality after coronary artery bypass grafting: a systematic review. Ann Thorac Surg 2009;87:1981-1992.

-32 Porter AG, Janicke RU: Emerging roles of caspase-3 in apoptosis. Cell Death Differ 1999;6:99-104.

-33 Haghikia A, Ricke-Hoch M, Stapel B, Gorst I, Hilfiker-Kleiner D: STAT3, a key regulator of cell-to-cell communication in the heart. Cardiovasc Res 2014;102:281-289.

34 Fischer P, Hilfiker-Kleiner D: Role of gp130-mediated signalling pathways in the heart and its impact on potential therapeutic aspects. Br J Pharmacol 2008;153 Suppl 1:S414-S427.

-35 McGaffin KR, Zou B, McTiernan CF, O’Donnell CP: Leptin attenuates cardiac apoptosis after chronic ischaemic injury. Cardiovasc Res 2009;83:313-324.

-36 Li Y, Takemura G, Okada H, Miyata S, Maruyama R, Li L, Higuchi M, Minatoguchi S, Fujiwara T, Fujiwara H: Reduction of inflammatory cytokine expression and oxidative damage by erythropoietin in chronic heart failure. Cardiovasc Res 2006;71:684-694.

37 Kodama H, Fukuda K, Pan J, Makino S, Sano M, Takahashi T, Hori S, Ogawa S: Biphasic activation of the JAK/ STAT pathway by angiotensin II in rat cardiomyocytes. Circ Res 1998;82:244-250.

-38 Levy DE, Lee CK: What does Stat3 do? J Clin Invest 2002;109:1143-1148.

39 Goodman MD, Koch SE, Fuller-Bicer GA, Butler KL: Regulating RISK: a role for JAK-STAT signaling in postconditioning? Am J Physiol Heart Circ Physiol 2008;295:H1649-H1656.

40 Pedretti S, Raddatz E: STAT3 $\alpha$ interacts with nuclear GSK3beta and cytoplasmic RISK pathway and stabilizes rhythm in the anoxic-reoxygenated embryonic heart. Basic Res Cardiol 2011;106:355-369.

41 Hausenloy DJ, Yellon DM: The mitochondrial permeability transition pore: its fundamental role in mediating cell death during ischaemia and reperfusion. J Mol Cell Cardiol 2003;35:339-341.

42 Boengler K, Hilfiker-Kleiner D, Heusch G, Schulz R: Inhibition of permeability transition pore opening by mitochondrial STAT3 and its role in myocardial ischemia/reperfusion. Basic Res Cardiol 2010;105:771785.

43 Gross GJ, Hsu A, Pfeiffer AW, Nithipatikom K: Roles of endothelial nitric oxide synthase (eNOS) and mitochondrial permeability transition pore (MPTP) in epoxyeicosatrienoic acid (EET)-induced cardioprotection against infarction in intact rat hearts. J Mol Cell Cardiol 2013;59:20-29.

44 Kensler TW, Wakabayashi N, Biswal S: Cell survival responses to environmental stresses via the Keap1Nrf2-ARE pathway. Annu Rev Pharmacol Toxicol 2007;47:89-116.

45 Wang Q, Zhang X, Ding Q, Hu B, Xie Y, Li X, Yang Q Xiong L: Limb remote postconditioning alleviates cerebral reperfusion injury through reactive oxygen species-mediated inhibition of delta protein kinase $\mathrm{C}$ in rats. Anesth Analg 2011;113:1180-1187.

-46 Wu ML, Ho YC, Yet SF: A central role of heme oxygenase-1 in cardiovascular protection. Antioxid Redox Signal 2011;15:1835-1846.

47 Downey JM, Omar B, Ooiwa H, McCord J: Superoxide dismutase therapy for myocardial ischemia. Free Radic Res Commun 1991;12-13 Pt 2:703-720.

48 Türei D, Papp D, Fazekas D, Földvári-Nagy L, Módos D, Lenti K, Csermely P, Korcsmáros T: NRF2-ome: an integrated web resource to discover protein interaction and regulatory networks of NRF2. Oxid Med Cell Longev 2013;2013:737591.

49 Liu SX, Zhang Y, Wang YF, Li XC, Xiang MX, Bian C, Chen P: Upregulation of heme oxygenase-1 expression by hydroxysafflor yellow A conferring protection from anoxia/reoxygenation-induced apoptosis in H9c2 cardiomyocytes. Int J Cardiol 2012;160:95-101.

50 Deng C, Sun Z, Tong G, Yi W, Ma L, Zhao B, Cheng L, Zhang J, Cao F, Yi D: $\alpha$-Lipoic acid reduces infarct size and preserves cardiac function in rat myocardial /reperfusion injury through activation of PI3K/Akt/Nrf2 pathway. Plos One 2013;8:e58371. 